\title{
Single cases promote knowledge transfer in the field of DBS
}

\author{
Markus Christen ${ }^{1 *}$ and Sabine Müller ${ }^{2}$ \\ 1 Institute of Biomedical Ethics, University of Zurich, Zürich, Switzerland \\ 2 Division of Mind and Brain Research, Department of Psychiatry and Psychotherapy, Charité - Universitaetsmedizin Berlin, Berlin, Germany \\ *Correspondence: christen@ethik.uzh.ch
}

The development and clinical application of deep brain stimulation (DBS) is based on the cooperation of various disciplines in order to address its neuroscientific, neurological, psychological, technological, and ethical challenges (Benabid et al., 2009). Safeguarding the quality of this interdisciplinary cooperation requires that novel issues (e.g., on adverse events) are raised timely in the scientific community and are communicated across disciplinary borders. In this opinion, we describe the development of the complexity of DBS research and assess knowledge transfer in terms of citations that transcend disciplinary borders. Our argument is based on an extended, ongoing meta-analysis of scientific journal papers on DBS in the nucleus subthalamicus (STN); a literature body that comprises more than 550 case reports, outcome studies, and review papers (Müller and Christen, 2011). Because STN-DBS, beginning in the early 1990s, is the most common DBS application, lessons learned in STN-DBS are important for evaluating clinical, societal, and ethical issues of novel DBS applications that emerge in psychiatry and other fields.

The complexity of DBS research is illustrated by the various issues that are discussed in the DBS literature. Our metaanalysis revealed a broad range of issues that can be grouped into four classes (see Figure 1A):

1. Understanding therapeutic effects: motor effects of DBS, effects on medication, and comparison with medication-based therapy, research about the physiological mechanisms of DBS.

2. Medical intervention issues: surgeryrelated issues (e.g., hemorrhage risks) and patient management (e.g., patient selection).

3. Affective, behavioral, and cognitive side effects of DBS: sequelae on behavior, cognition, mood, language, and quality of life.
4. Other issues: cost-benefit studies, technological issues (e.g., battery life), effects of DBS on autonomous functions, sensory systems, emotion recognition, sleep, and body weight.

For investigating how and when the discussion in the scientific communication about those issues has developed, the analysis of conference posters is preferable to the analysis of journal papers, since posters are published faster, have a lower publication threshold, and are more thematically focused. The International Congress of Parkinson's Disease and Movement Disorders (since 1990 biannually, since 2004 annually) maps very accurately the research on DBS applications for movement disorders. Since the amount of DBS journal papers has significantly increased after 2000 - indicating a transition from a "pioneer phase" to an "application phase" (Müller and Christen, 2011), we compare the posters published on the 2002 (175 DBS posters out of 1,183) and 2010 (124 DBS posters out of 1,067) conferences. Thus we cover a time span in which the scientific debate on DBS issues already was established. A content analysis of the posters presented in 2002 reveals a dominance of issues about understanding the therapeutic effects of DBS. However, the thematic spectrum was impressively wide and broadened further in 2010, where in particular the fractions of posters about patient management and about behavioral and cognitive side effects have increased.

One way to investigate knowledge transfer between different disciplines that cooperate for developing new therapies is an impact analysis, i.e., a comparison of the number of publications with the number of citations of those publications in different disciplinary fields (Christen, 2008). In the Thomson Reuters Web of Knowledge database (the broadest academic citation indexing and search service), each publication is related to one or several subject categories based on the journal in which it has been published. These subject categories are pooled to "disciplinary clusters," whereas the pooling is adapted to the type of problem and the number of citations obtained. By way of example, if $50 \%$ of the publications of a specified literature body are attributed to one cluster, whereas only $10 \%$ of all citations generated by this literature body are attributed to the same cluster, a publication-citation-transfer between this cluster and other clusters has happened.

For the impact analysis, we have compared six disciplinary clusters of three subsets of publications from our DBS literature body (see below) with the set of publications that cite these publications. The impact analysis shows that the subject category "clinical neurology" covered the most citations (and publications), and thus became a separate cluster, whereas the other disciplinary clusters (Neuroscience, Biology, Psychiatry/Psychology, Medicine, Social/Technical Sciences and Humanities) were formed by several subject categories with thematic similarities. Subject categories of technical and social sciences or humanities obtained only a few citations and were therefore pooled in one large cluster. It has to be noted that journals for social sciences and humanities are covered insufficiently by the Web of Knowledge database. Therefore the impact analysis underestimates the impact of the DBS literature in these disciplines.

The following publication-subsets from our STN-DBS literature body ( 550 publications) have been chosen for the impact analysis: 40 Case Reports on adverse events after STN-DBS; 44 Outcome Studies that achieve high "relevance ratings" (i.e., they have been regularly analyzed by the DBS-review-papers that form the third set); 23 "high quality" Review Papers that used a standard meta-analysis methodology or were based at least on a systematic 

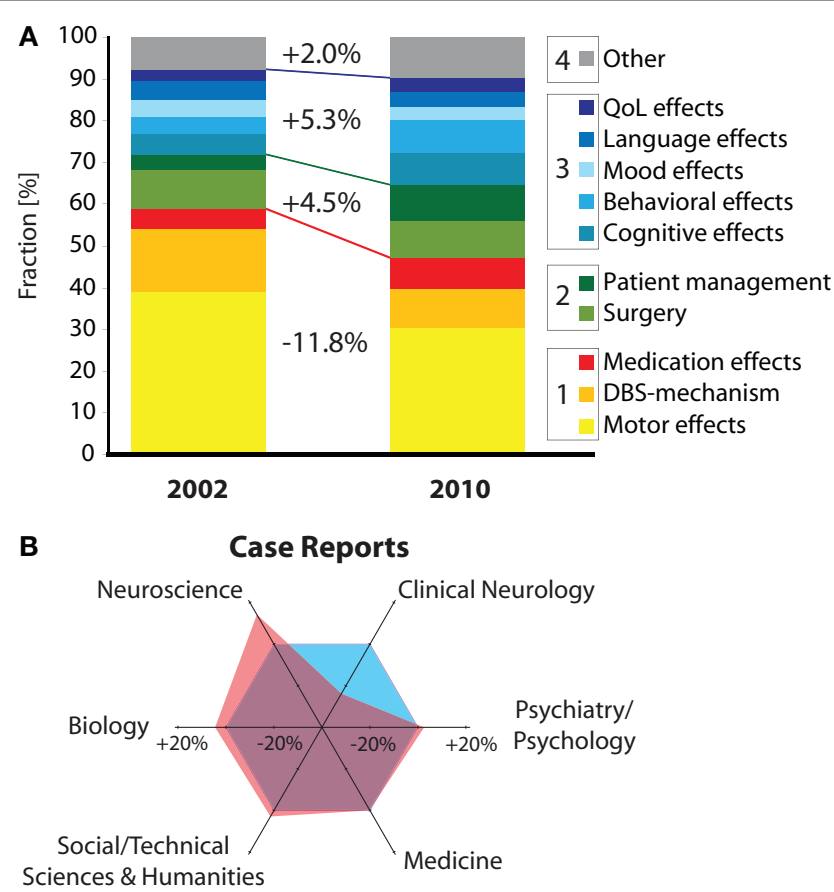

\section{Outcome Studies}

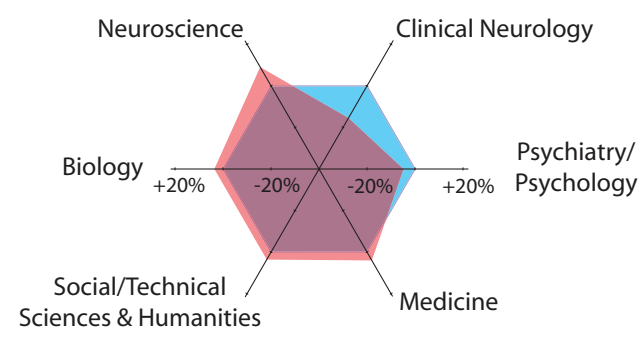

\section{Review Papers}

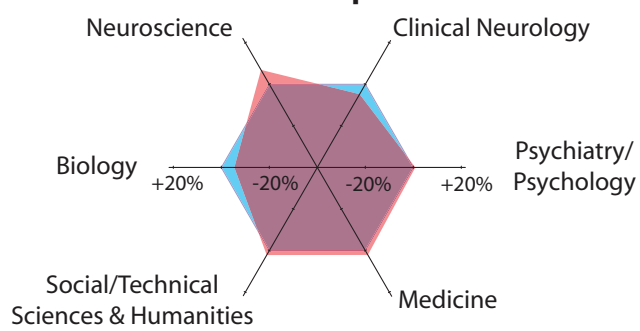

FIGURE 1 | (A) Change of the relative weight of issues discussed in the DBS community based on an analysis of the posters published in 2002 and 2010 on the International Congress of Parkinson's Disease and Movement Disorders. The issues are grouped into four classes: 1. Understanding therapeutic effects, 2. Medical intervention issues, 3. Affective, behavioral, and cognitive side effects of DBS, 4. Other issues. (B) Impact analysis for three types of DBS literature: case reports, outcome studies, and review papers. The blue hexagon is the reference (percentage of publications equals percentage of citations in each cluster), whereas the red polygon shows the actual fraction of citations minus the fraction of publications for each disciplinary cluster.

examination of outcome studies (i.e., no narrative reviews). Figure 1B shows the results of the impact analysis for each type in a spider diagram that displays the citation fraction minus the publication fraction for each cluster in percent. The blue hexagon is the reference (i.e., the fraction of citations minus the fraction of publications equals zero), whereas the red area shows the actual differences of the fractions of citations minus publications. In all three cases, the cluster "Clinical Neurology" was the main source of publication-citationtransfers. However, the "overall transfer" - i.e., the sum of all positive (or all negative) transfers between all clusters - differ markedly between the three types of publications: Whereas in the case reports $23.8 \%$ of all citations were generated outside their disciplinary origin, only $10.9 \%$ of citations generated by review papers left their disciplinary origin (outcome studies: $20.5 \%)$. The cluster "Neuroscience" was the main transfer target. In absolute numbers, only few citations (Case Reports: 2.9\%, Outcome Studies: 3.7\%, Review Papers: $2.0 \%$ ) were generated from the cluster "Social/Technical Sciences and Humanities."

In summary, two lessons can be learned: First, the DBS community indeed recognized the complexity associated with this novel therapeutic approach and adapted its focus to emerging issues. Second, not high quality review papers, but reports on complex, single cases spearheaded the interdisciplinary knowledge transfer. Therefore the discussion about clinical, societal, and ethical issues of DBS should not rely on the assumption that the DBS community underestimates the complexity of DBS. Further research should focus on the question how reports on paradigmatic cases diffuse into different disciplines in order to understand the communication processes that accompany the development of novel, stimulation-based therapies for psychiatric and other diseases.

\section{REFERENCES}

Benabid, A. L., Chabardes, S., Mitrofanis, J., and Pollak, P. (2009). Deep brain stimulation of the subthalamic nucleus for the treatment of Parkinson's disease. Lancet Neurol. 8, 67-81.

Christen, M. (2008). Varieties of publication patterns in neuroscience at the cognitive turn. J. Hist. Neurosci. 17, 207-225.

Müller, S., and Christen, M. (2011). Deep brain stimulation in Parkinsonian patients - ethical evaluation of cognitive, affective and behavioral sequelae. $A J O B$ Neurosci. 2, 3-13.

Received: 12 January 2011; accepted: 18 April 2011; published online: 05 May 2011.

Citation: Christen Mand Müller S (2011) Single cases promote knowledge transfer in the field of DBS. Front. Integr. Neurosi. 5:13. doi: 10.3389/fnint.2011.00013

Copyright (C) 2011 Christen and Müller. This is an openaccess article subject to a non-exclusive license between the authors and Frontiers Media SA, which permits use, distribution and reproduction in other forums, provided the original authors and source are credited and other Frontiers conditions are complied with. 